\title{
Modelling of MOFs for energy and environment-related applications
}

\author{
Guilllaume Maurin $^{1}$ \\ ${ }^{1}$ Université Montpellier, Montpellier, France \\ E-mail: gmaurin@um2.fr
}

Molecular simulations have largely contributed to the emergence of Metal Organic Frameworks (MOFs) not only for the resolution of the crystal structures of the most complex and poorly crystallized solids but also to enumerate all the plausible structures constructed by the assembly of a large diversity of inorganic and organic building blocks. Besides this in silico design of novel MOFs which has been only rarely validated so far by the post-synthesis of the desired material, a computational effort has been deployed to modulate the chemical and topological features of existing architectures specifically targeted for societally-relevant applications. Molecular modelling has been also frequently used to guide interpretation of the experimental data by providing a deep understanding of the microscopic adsorption/separation mechanism with the objective to drive the synthesis effort towards tuned materials with the required features for an optimization of their properties. This presentation will highlight the invaluable contribution of the computational approaches from the birth of novel MOFs and their structure elucidations to the characterization and understanding of their properties, throughout recent advances our groups have made in this field. A special emphasizes will be devoted to a series of recent MOFs that show promising adsorption/separation performances for natural gas upgrading, carbon capture and interesting features for mechanical energy storage and proton conduction.

J.W. Yoon, H. Chang, S-J Lee, Y.K. Hwang, D-Y Hong, S-K Lee, S. Jang, T-U Yoon, K. Kwac, Y. Jung, R.S. Pillai, F. Faucher, A. Vimont, M. Daturi, G. Férey, C. Serre, G. Maurin, Y-S Bae, J.S. Chang, Nature Materials, DOI: 10.1038/nmat4825

S. Krause, V. Bon, I. Senkovska, U. Stoeck, D. Wallacher, D. Tobbens, S. Zander, R.S. Pillai, G. Maurin, F.X. Coudert, S. Kaskel, Nature, 532, 348, 2016.

Mechanical energy storage performance of an aluminum fumarate metal-organic framework, P. G. Yot, L. Vanduyfhuys, E. Alvarez, J. Rodriguez, J.P. Itié, P. Fabry, N.Guillou, T. Devic, I. Beurroies, P. L. Llewellyn, V. Van Speybroeck, C.Serre and G. Maurin, Chem. Sci, 7, 446, 2016.

Keywords: microporous and mesoporous MOFs, gas capture/separation, mechanical energy 